\title{
EFFECT OF METRONIDAZOLE LOADED NANOPARTICLES ON TREATMENT OF INDUCED PERIODONTAL DEFECTS IN DOGS
}

\author{
Enas Ahmed Elgendy*, Amel Mohamed Ezzat Abd-Elhamid**, \\ Alaa M. Metwalli Moustafa*** and Tamer Elamer Abo Shady****
}

\begin{abstract}
Objective: The aim of the present study was to compare between $25 \%$ metronidazole gel and $25 \%$ metronidazole loaded nanoparticle gel formulation on healing of experimentally induced periodontal defects in dogs.

Materials and Methods: Periodontal defects were induced by ligatures placement in 10 beagle dogs in both lower sites. The defects were randomly and equally allocated in a split-mouth design to either Metronidazole gel nanoparticles gel (25\%) (Experimental site group I) or Metronidazole gel $(25 \%)$ (Control site group II) The animals were sacrificed with an overdose of anesthesia at 8 weeks and block sections of the defects were collected for histological and histometric analysis.

Results: The results showed improved outcomes in terms of bone as well as new cementum and functionally oriented periodontal ligament formation. However, sites treated with metronidazole loaded nanoparticles $(25 \%)$ gel formula (experimental group I) showed significantly greater regenerative potential of the attachment apparatus in terms of newly formed cementum and bone compared to conventional metronidazole gel treated group.
\end{abstract}

Conclusion: This, in turn, proves that $25 \%$ metronidazole loaded nanoparticle gel is more efficient compared to conventional metronidazole gel in treatment of periodontal defects.

KEY WORDS: Metronidazole loaded nanoparticle. Metronidazole gel, Induced Periodontal defects.

\section{CLINICAL RELEVANCE:}

Scientific Rationale for Study: Evaluating the effect of metronidazole loaded nanoparticles on the treatment of induced periodontal defects in dogs. Principal Findings: Findings from this animal study of 10 beagle dogs supported the potential of metronidazole loaded nanoparticles gel, as compared to $25 \%$ metronidazole gel, for providing a safe, inexpensive, and effective therapy for induced periodontitis by regenerating new bone as well as new cementum and forming functionally oriented periodontal ligaments. Practical Implications: Metronidazole loaded nanoparticles gel can be used as adjunctive treatment of periodontitis.

\footnotetext{
* Associate Professor of Oral Medicine, Periodontology and Oral Diagnosis. Faculty of Dentistry, Kafr El-Sheikh University. Egypt.. ** Assistant Professor of Oral Biology, Dental Faculty, Tanta University. Egypt.

*** Assistant Professor of Surgery, Anesthesia and Radiology, Faculty of Veterinary Medicine, Kafr El-Sheikh University, Egypt. **** Lecturer of Oral Medicine, Periodontology and Oral Diagnosis. Faculty of Dentistry, Tanta University. Egypt.
} 


\section{INTRODUCTION}

Periodontitis is characterized by destruction of the periodontal ligament, a resorption of the alveolar bone and the migration of the junctional epithelium along the tooth surface. Periodontal diseases are caused by pathogenic bacterial species that adhere to dental surfaces forming complex communities, known as oral biofilms. From more than 700 different species identified in the oral microbiota, only a small group of 10-15 species are considered to be significantly associated with the initiation and progression of periodontitis ${ }^{[1]}$. The various periodontal pathogens which are susceptible in the disease progression includes Aggregatibacter actinomycetemcomitans, Porphyromonas gingivalis, Prevotella intermedia, Tannerella forsythia, Campylobacter rectus, and Treponema denticola ${ }^{[2]}$.

The main goal of periodontal therapy has been focusing on the reduction or eradication of specific periodontal pathogens and ensuring that periodontal pockets are free of granulation tissue for proper healing to occur. Mechanical debridement alone may fall to reduce or eliminate anaerobic infection at the base of the pocket. The adjunctive use of antimicrobials has been indicated to improve the clinical outcomes in periodontal conditions, which can be used either systemically or locally ${ }^{[3]}$.

Local delivery of antimicrobials in periodontal pockets may be carried out with fibers, films, microparticles and gels made of biodegradable or non-degradable polymers and have been proposed as effective methods to administer antimicrobial agents in periodontal therapy ${ }^{[4]}$.

Among the antimicrobials used for the treatment of periodontitis, metronidazole is particularly suitable due to its restricted spectrum of activity against obligate anaerobes such as Prphyromonas gingivalis and Prevotella intermedia and its limited side effects ${ }^{[5]}$. Metronidazole is bactericidal to anaerobic organisms and is believed to disrupt bacterial DNA synthesis ${ }^{[2]}$.
Nanoparticles have received considerable attention in recent years due to their wide range of applications in various fields such as drug delivery systems ${ }^{[6,7]}$. Drug-delivery systems can be synthesized with controlled composition, shape, size and morphology of nanoparticles. Their surface properties can be manipulated to increase solubility, immuno-compatibility and cellular uptake ${ }^{[8]}$.

The present study was carried out to compare between the metronidazole and metronidazole loaded nanoparticles on treatment of induced periodontitis in dogs.

\section{MATERIALS AND METHODS}

\section{Gel preparation}

\section{The ingredients and preparation of (25\%) Metro- nidazole gel formulation}

Metronidazole gel was prepared in the Faulty of Pharmacy laboratory, Delta University, Egypt by the method stated by Ramadan et al., ${ }^{[9]}$. Metronidazolebenzoate was supplied by Alexandria Company for pharmaceuticals, Egypt. First, 0.50 gram poly lactide-co-glycolide (PLGA) was placed in a glass vial then, triacetin $4.25 \mathrm{~g}$ was added, heated at $60^{\circ}$ $\mathrm{C}$, and agitated using a mechanical shaker overnight to obtain a clear solution. Afterthat, the drug was dissolved in propylene glycol, added to the polymer solution and sonicated for 30 seconds to get a uniform dispersion. When PLGA solution came into contact with the in vivo aqueous environment, it transformed to a solid matrix that released the loaded drug for a prolonged period of time.

\section{Preparation of (25\%) Metronidazole loaded nanoparticles gel formulation}

The encapsulation of the drug into a biodegradable polymer PLGA were done and purchased from the nanotech company for photoelectronics, Dreamland, El Wahat road, 6 October City). Oil in water $(\mathrm{O} / \mathrm{W})$ was used to prepare the 
metronidazole loaded PLGA nanoparticles. The oil phase was created by dissolving PLGA in acetone at room temperature. Then metronidazole was added to poly vinyl alcohol $(30 \mathrm{kDa})$ solution to prepare the water phase. The oil phase was pipetted into the water phase drowsily and homogenized (Ultraturrax, IKA, Germany) at $8000 \mathrm{rpm}$ for 5 minutes. After evaporation, nanoparticle were collected by centrifugation and washed three times to remove nonencapsulated drug. Finally gelatinous solution was obtained. Metronidazole gel $(25 \%)$ and Metronidazole gel nanoparticles gel $(25 \%)$ would be done and purchased from the nanotech company for photo- electronics (Dreamland, El Wahat road, 6 October City).

\section{Experimentally induced periodontitis}

Animals: A total of 10 adult male mongrel dogs weighing 20-25 kg were selected from Surgery, Anesthesiology and Radiology Department, Faculty of veterinary Medicine, Khafr Elshikh University to be used in this study. The study protocol was reviewed and approved by the Animal Care Committee, Faculty of Veterinary Medicine, Kafrelsheikh University, in accordance with Egyptian ethical codes for studies on experimental animals. The animal had intact dentition with healthy periodontium. All experimental operations would be performed with dogs under general anesthesia. To prepare the healthy gingiva, all teeth would be scaled with hand instruments. The animals were fed a soft diet throughout the study to reduce the chance of mechanical interference with healing during food intake ${ }^{[10]}$.

The animals were anaesthetized using $0.2 \%$ ketamine $\mathrm{HCl}$ by intravenous drip method after premedication with xylazine $(5 \mathrm{mg} / \mathrm{kg})$.The surgical sites were disinfected with $10 \%$ povidine-iodine solution $1 \%$ titratable iodine. After mucoperiosteal flap elevation on the mandibular third (or fourth) premolars of each dog, shallow grooves would be made in the mesial and distal region of each tooth with a round bur to facilitate the insertion of ligature. Ligature $2 \mathrm{~mm}$ in diameter was inserted around the cervical region of the tooth and then suture the flap (Figure1a, 1b, 1c). For pain control, tramadol (4 $\mathrm{mg} / \mathrm{kg}$, intramuscular injection) would be administered twice a day for 3 days after placing the ligatures. For the next sixty days, the experimental dogs received soft-moistened food to encourage plaque formation. Meanwhile, the ligatures would be checked daily to ensure no ligature fell out. Two months later, the animals were anaesthetized using $0.2 \%$ ketamine $\mathrm{HCl}$ by intravenous drip method after premedication with xylazine $(5 \mathrm{mg} / \mathrm{kg})$. The surgical sites were disinfected with $10 \%$ povidineiodine solution $1 \%$ titratable iodine. Buccal mucoperiosteal flaps were elevated, and the buccal bone was removed (surgically defect created) with burs in the buccal aspect of second premolars and third bilaterally premolars (split mouth study). Two reference notches were created with a round bur on the root surfaces one at crest of the alveolar bone (N1) and another one at the base of the defect (N2). The root surfaces were carefully scaled, planned, flushed with sterile saline, and then dried with sterile gauze. Each defect underwent randomly one of two treatment modalities: Metronidazole gel nanoparticles gel (25\%) (Experimental site group I) or Metronidazole gel (25\%) (Control site group II) and then suture the flap (Figure 1d, 1e, 1f). All of the surgical procedures were done by the same clinicians.

\section{Postoperative care}

After the operative procedures the dogs were received Acupan (I.M every 12 hours) for pain control and intramuscular administration of antibiotics, Tetracycline HCL (Terramycine retard $125 \mathrm{mg}$ intramuscular) in the first two days. After that tetracycline HCL was given by mixing it with the dog's food for 7 days. The dogs were kept in separate cages, and a daily topical application of $0.12 \% \mathrm{CHX}$ solution during the healing period was performed. The animals were observed daily until suture removal and at least twice weekly thereafter. 


\section{Histological examination}

Two months post-surgically the animals were sacrificed using an overdose of anesthesia for histological examinations. The jaws were dissected and the blocks containing the experimental specimens were obtained (premolars segment). The blocks were fixed in $10 \%$ neutral formalin solution for one week. Blocks biopsy specimens were transferred to $10 \%$ EDTA solution containing $5 \%$ sodium sulfide until demineralization occurred. The decalcified specimens were washed in running water, dehydrated and embedded in paraffin wax (the paraffin wax melting point should not exceed more than $56^{\circ} \mathrm{c}$ to avoid the burning of the tissues) for 24 hours and then embedded in paraffin blocks. All formalin-fixed-paraffin-embedded tissues were serially cut bucco-lingualy with a rotary microtome at $5 \mu \mathrm{m}$ thickness. The sections were then placed on glass slides and incubated overnight at $60^{\circ} \mathrm{C}$ after which they were rehydrated in xylene, rinsed in ethanol and then water. The slides were stained with basic hematoxylin stain and counterstained with eosin for contrast and evaluation. The 2 notches described previously were used as reference points in the histological examination.

\section{Histometric analysis}

The histometric analysis included the study of 10 sections in each group. The histological hematoxylin and eosin stained sections were photographed and digitized with a light microscope coupled to a digital camera that was connected to a monitor and a personal computer, using the crest of the alveolar bone and the root notch as reference points.

\section{The histometric parameters were}

1. The Defect Height (DH): the distance between the base of the coronal notch (alveolar crest) and the base of the apical notch in millimeters.

2. Junctional Epithelial Migration (JE): the distance from the base of the coronal notch (alveolar bone crest) to the apical extension of the junctional epithelium.

3. Connective Tissue Adhesion (CT): the distance from the apical extension of the junctional epithelium to the coronal extension of the new cementum.

4. Cementum Regeneration (NC): the distance from the base of the apical notch to the coronal extension of newly formed cementum on the root surface.

5. Alveolar Bone Regeneration (NB): the distance from the base of the apical notch to the coronal extension of the newly formed alveolar bone.

All of the histopathological and histometric analysis were done by the same histopathologist who was masked to the treatment rendered.

\section{Statistical analysis}

All the results were tabulated and statistically analyzed using computer software named the Statistical Package for Social Science (SPSS version 16). Data in the present study were presented as mean and standard deviation (SD). The comparisons between the study groups were determined by using an independent-samples t-test, and the level of significance was established at a value of $\mathrm{P}<0.05$.

\section{RESULTS}

\section{Histological observations}

During the experimental period, all dogs remained healthy with normal behavior and consumption of food and water. Post insertion of infected ligatures around the cervical region of the tooth, suppuration formation was observed at the site of the surgical procedures.

After two months, the histological findings in group I (Metronidazole Loaded Nanoparticles) revealed complete healing of the periodontium that demonstrated similar features to native periodontium. 
Coronal to the root notch (N2), new attachment along the created bony defect was found, in addition to, newly formed bone and osteoblastic cells which were arranged along the bone marrow space. No migration of junctional epithelium was observed.
Moreover, dense well organized highly cellular periodontal ligament fibers oriented perpendicular to the tooth surface was found to be inserted into the newly formed thick layer of cementum and bone (Figure 2).

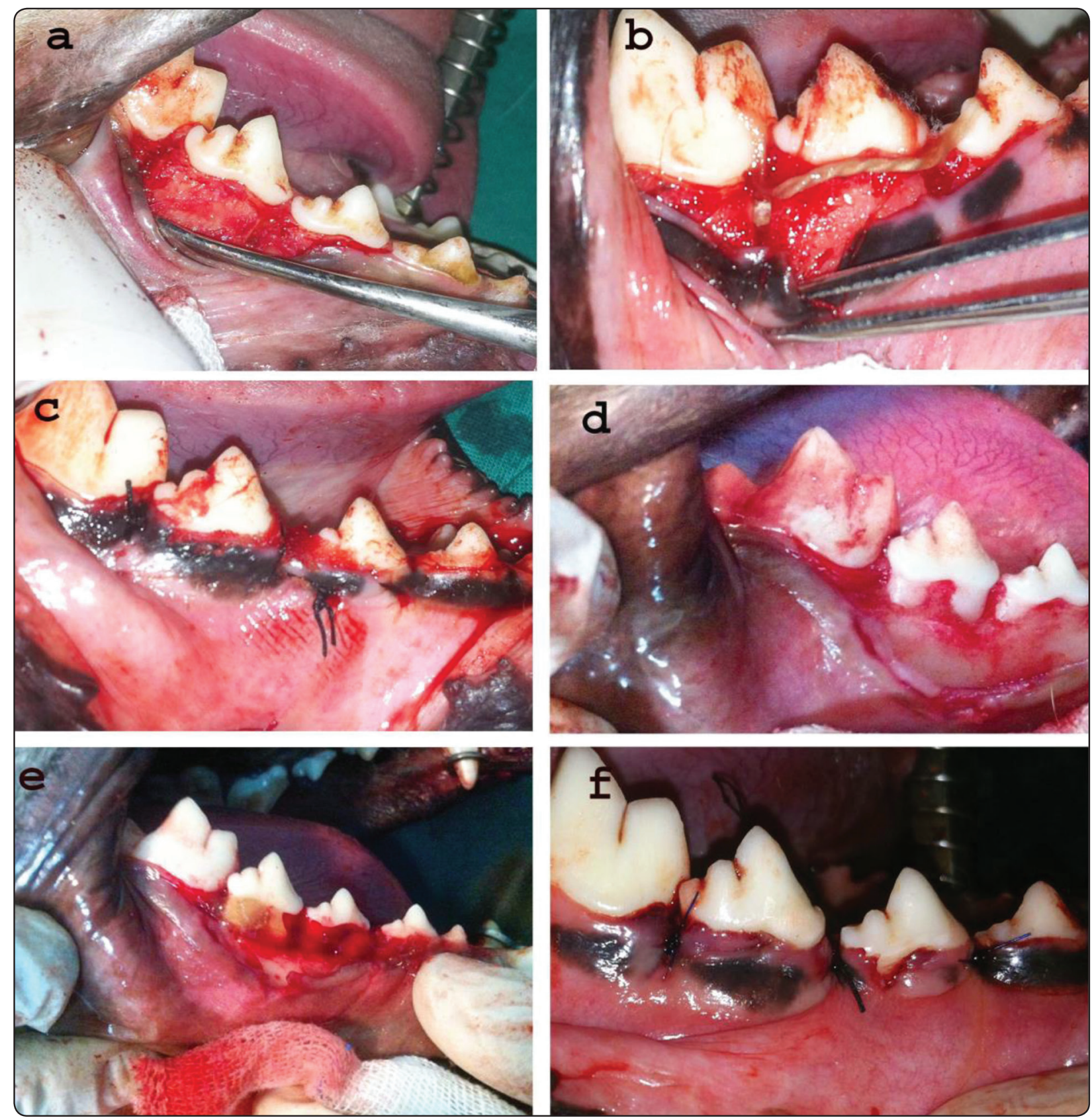

Fig. (1) Clinical photographs showing a) premolars area following flap reflection, b) Ligature placement, c) Mucoperiosteal flap sutured, d) Experimentally induced periodontitis and surgically created defect, e) Local application of the metronidazole gel,f) Mucoperiosteal flap sutured. 

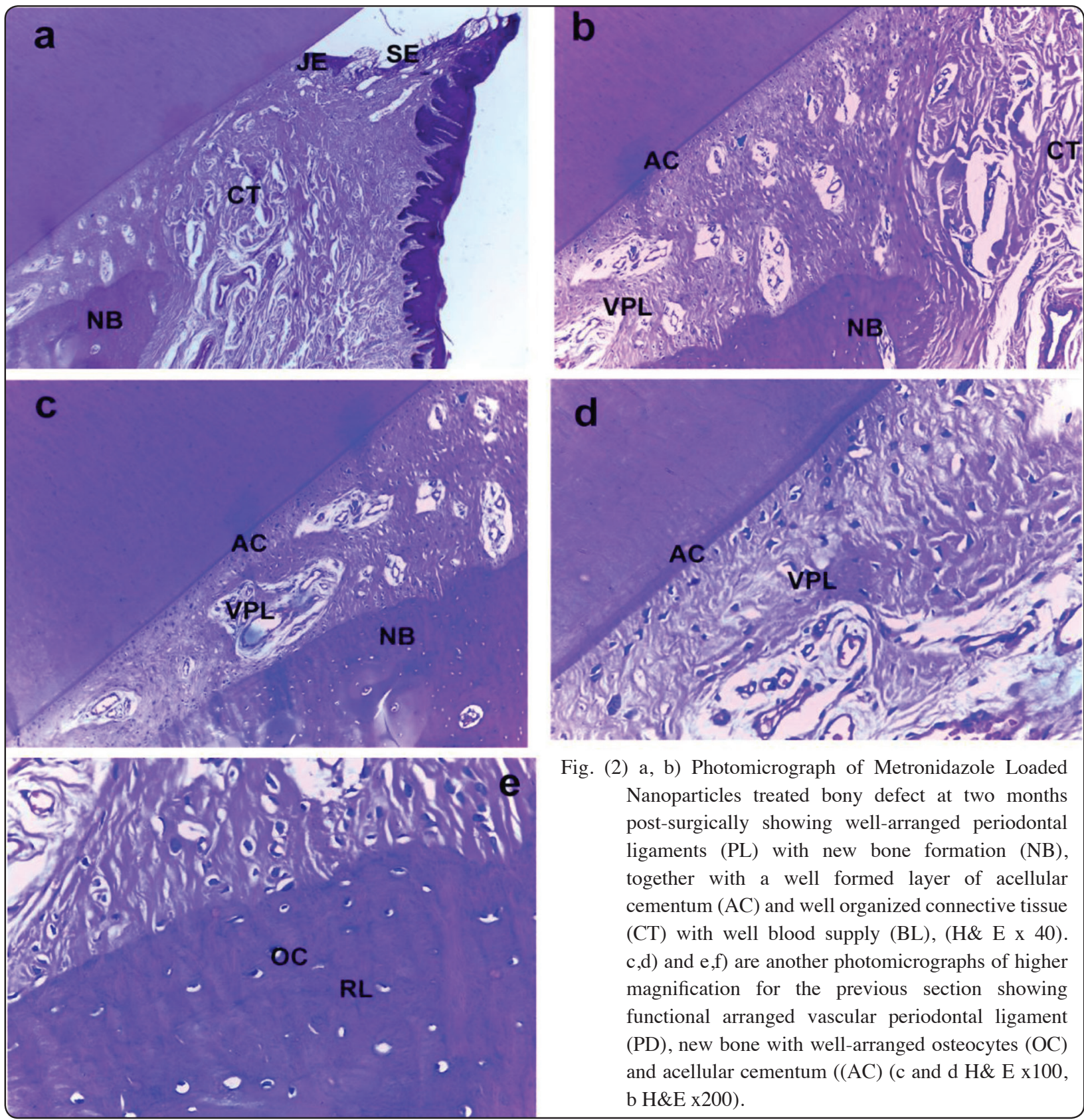

Fig. (2) a, b) Photomicrograph of Metronidazole Loaded Nanoparticles treated bony defect at two months post-surgically showing well-arranged periodontal ligaments (PL) with new bone formation (NB), together with a well formed layer of acellular cementum (AC) and well organized connective tissue (CT) with well blood supply (BL), (H\& E x 40). $\mathrm{c}, \mathrm{d})$ and $\mathrm{e}, \mathrm{f})$ are another photomicrographs of higher magnification for the previous section showing functional arranged vascular periodontal ligament (PD), new bone with well-arranged osteocytes (OC) and acellular cementum ((AC) (c and d H\& E x100, b H\&E x200).

In group II, H\&E stained section showed junctional epithelium ending apical to the notch at the original gingival margin (N1). Furthermore, bundles of non-inflamed connective tissue extended from the end of junctional epithelium parallel to tooth surface. Finally, well organized highly cellular periodontal ligament fibers perpendicular to the tooth surface was found to be inserted into the newly formed cementum and new bone coronal to notch (N2) and was delineated by reverse line (Figure 3).

\section{Histometric analysis}

The metronidazole group and metronidazole loaded nanoparticles group were compared according to the junction epithelial migration (JE), connective tissue adhesion (CT), new cementum (NC), and new bone (NB) formation. The groups were compared by the independent-samples t-test. The two treatment groups showed no significant differences regarding the Defect height, JE and CT. 


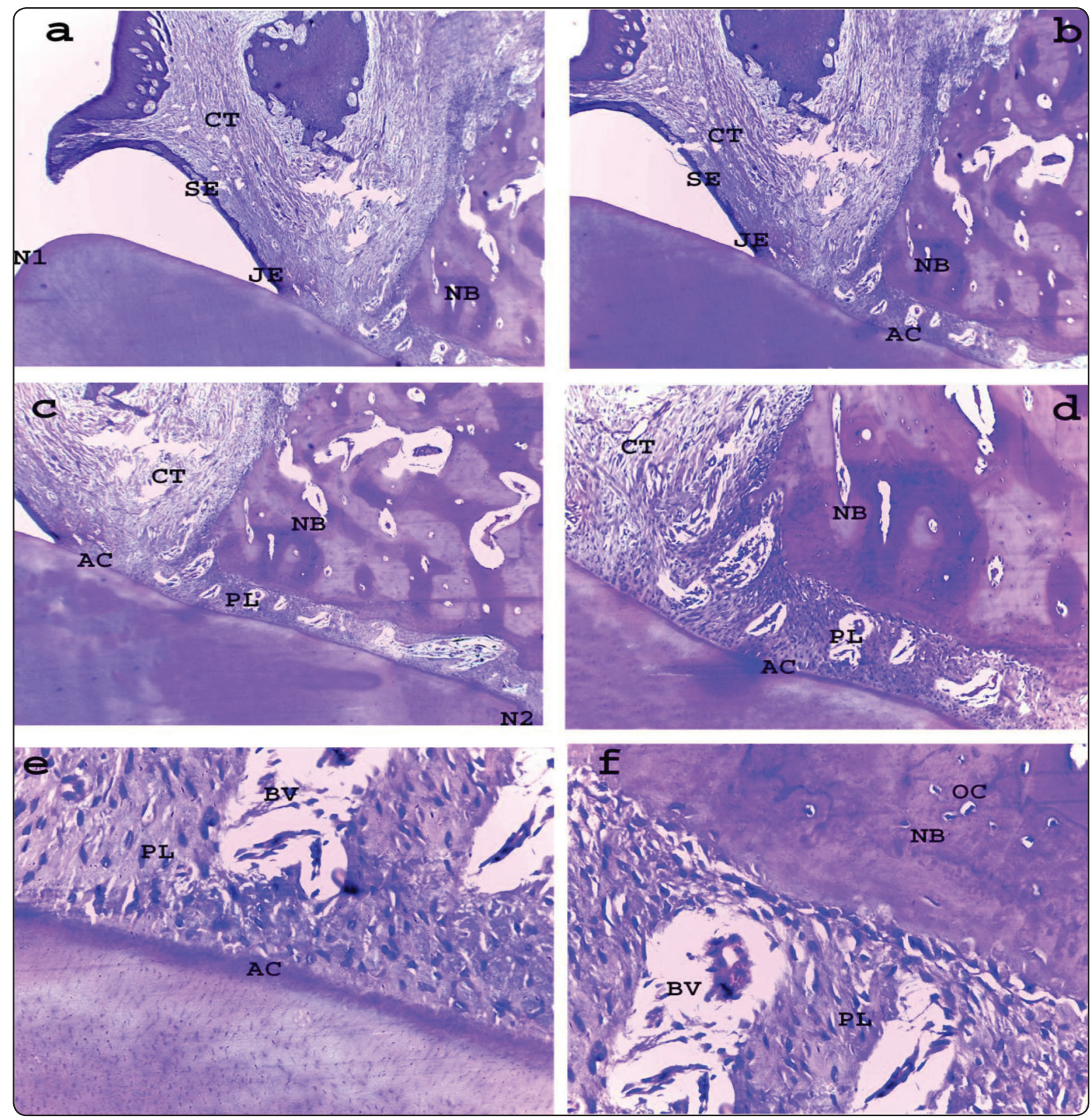

Fig. (3): a, b Photomicrographs of metronidazole gel treated bony defect two months post-surgically showing junction epithelium (JE) extending below the level of notch (N1) with new bone formation (NB), layer of acellular cementum (AC) and vascular connective tissue (CT) (H\&E x 40). While c, d \& e, Another photomicrographs of higher magnification for the previous section showing arrangement of periodontal ligament (PD) with some granulation tissue associatedwith some vascular blood supply (BV), new bone (NB) and Acellular cementum ((AC) (H\& E x100 and d H\&E x200).

Data showed that the mean difference of NB and $\mathrm{NC}$ were statistically significant $(\mathrm{P}<0.05)$ between all treated groups. The new bone formation and new NC was greater in the metronidazole loaded nanoparticles group in comparison with the metronidazole group (Table 1). 
TABLE (1) Histometric analysis of the measured parameters in group I (metronidazole Loaded Nanoparticles gel) and group II (metronidazole gel) presented as mean \pm SD.

\begin{tabular}{|c|c|c|c|}
\hline Tested parameters & $\begin{array}{c}\text { Group I } \\
\mathrm{N}=\mathbf{1 0} \\
\text { Mean }(\mathbf{m m}) \pm \mathrm{SD}\end{array}$ & $\begin{array}{c}\text { Group II } \\
\mathrm{N}=\mathbf{1 0} \\
\text { Mean }(\mathrm{mm}) \pm \mathrm{SD}\end{array}$ & $\begin{array}{c}\text { t-test } \\
\text { P-value }\end{array}$ \\
\hline Defect height & $4.94 \pm 0.46$ & $4.70 \pm 0.58$ & $\begin{array}{l}0.999 \\
0.332\end{array}$ \\
\hline Junction epithelial migration & $0.56 \pm 0.07$ & $0.65 \pm 0.12$ & $\begin{array}{l}1.739 \\
0.100\end{array}$ \\
\hline Connective tissue adhesion & $0.96 \pm 0.14$ & $1.01 \pm 0.11$ & $\begin{array}{l}0.743 \\
0.468\end{array}$ \\
\hline New cementum & $3.72 \pm 0.36$ & $3.37 \pm 0.34$ & $\begin{array}{c}2.152 \\
0.046^{*}\end{array}$ \\
\hline New bone & $3.62 \pm 0.33$ & $3.10 \pm 0.21$ & $\begin{array}{c}4.149 \\
0.001 *\end{array}$ \\
\hline
\end{tabular}

Group I: Metronidazole Loaded Nanoparticles treated bony defect

Group II: Metronidazole gel treated bony defect

* Significant difference between the two groups at two months follow-up period $(P<0.05)$

\section{DISCUSSION}

The ultimate goal in periodontal therapy is regeneration. The establishment of a new connective tissue attachment with fibers inserted into new bone and new cementum on a previously diseased root surface is the ideal objective ${ }^{[11]}$.

Dogs were selected as an experimental model in order to ascertain the type of attachment of the treated defect, since block sections in humans would be both unethical and unacceptable by most patients. Moreover, the features of periodontitis in dogs and other non-human primates, most closely resemble the features of the disease in humans, because they are closely related to humans phylogenetically, and the anatomy and physiology of their teeth and periodontium are also similar. In addition, the large tooth size allows for adequate surgical access and the treatment of periodontal lesions ${ }^{[12]}$.

In the current study, healing of the periodontal defects was assessed after 8 weeks in dogs to evaluate and compare the regenerative response induced by the tested materials, metronidazole and metronidazole nanoparticles. Several histological studies reported that an 8-week healing interval was required to evaluate periodontal bone regeneration and there was no significant difference in bone regeneration between an 8 and 24-weeks interval ${ }^{[13-16]}$. Therefore, in our study, it was decided that an 8-week healing period was a sufficient period to observe the wound healing process.

The placement of 2 reference notches was useful, where the gingival notch (N1) represented the exact location of the pre-operative gingival margin and the bone notch (N2) represented the end of the defect. Therefore, any connective tissue attachment observed in postsurgical examination coronal to $\mathrm{N} 2$ was considered new connective tissue attachment.

In dentistry, drug-loaded nano-pharmaceuticals have been extensively utilized over the past few years and are studied in almost all dental related fields. In the current study poly lactide-co-glycolides (PLGAs) was used to control the release of the 
treatment drug as it is not only biocompatible but also a biodegradable polymer that is hydrolytically degraded into nontoxic oligomers or monomers, lactic acid and glycolic acid. Moreover, owing to PLGA, sustained drug release was allowed within the target site over a period of days to weeks and thus increasing the therapeutic benefit, along with minimizing side effects ${ }^{[17-21]}$.

Metronidazole was selected in the current study since it has antimicrobials effects against obligate anaerobes by inhibiting DNA synthesis. It is known to be converted into a reactive reduced form that affects specifically anaerobic rods and spirochetes in subgingival microflora ${ }^{[22]}$.

Histological results at 2 months in the current study, revealed there was dense well organized highly cellular periodontal ligament fibers perpendicular to the tooth surface inserted into the newly formed layer of cementum and new bone coronal to notch (N2) in both tested groups. These results could be attributed to the antimicrobial effect of metronidazole gel and these results are in accordance with Walker ${ }^{[23]}$,

Poulet et al., ${ }^{[24]}$, and Bahar et al., ${ }^{[25]}$ who found that black-pigmented anaerobe $P$. gingivalis were highly susceptible to metronidazole.

Meanwhile, the histomorphometric findings revealed that defects treated with metronidazole loaded nanoparticles showed better healing. To elaborate, group I showed a more significant increase in new bone and cementum formation as compared to group II $(\mathrm{P}<0.05)$. These results could be attributed to enhanced properties of nanostructured metronidazole gel including high stability (i.e., long shelf life); high carrier capacity (i.e., many drug molecules can be incorporated in the particle matrix); ${ }^{[26]}$ enhanced delivery or uptake by, target cells; ${ }^{[27]}$ high penetration of area, which may be inaccessible to other delivery system, due to its small size. It has been established that nanocarriers can become concentrated preferentially to tumors and inflammatory sites, and at antigen sampling sites by virtue of the enhanced permeability and retention (EPR) effect of the vasculature ${ }^{[28]}$.

\section{CONCLUSIONS}

Based on the results of the present study, it could be concluded that the metronidazole loaded nanoparticles $25 \%$ had an additive effect on the histological outcome in the form of more cementum and bone formation when compared to conventional metronidazole gel.

\section{RECOMMENDATION}

Further studies to assess whether metronidazole loaded nanoparticles gel $25 \%$ could be used as an adjunct to conventional and surgical periodontal therapy in human.

\section{REFERENCES}

1. Lazăr L, Monea M, Biriş Carmen. Metronidazole Dental Gel as an Adjunct to Scaling and Root Planning: A Clinical Study. Acta Medica Transilvanica 2014;2:291-293.

2. Rajagopalan A, Thomas JT. Effectiveness of Metronidazole as Local Drug Delivery in Periodontal Diseases - A Review. IOSR Journal of Dental and Medical Sciences. 2014;13:25-28.

3. Herrera D, Matesanz P, Bascones-Martínez A, Sanz M. Local and systemic antimicrobial therapy in periodontics. J Evid Based Dent Pract. 2012;12:50-60.

4. Hanes PJ, Purvis JP. Local anti-infective therapy: pharmacological agents. A systematic review. Ann Periodontol.2003;8:79-98.

5. Mitchell DA. Metronidazole: its use in clinical dentistry. $\mathrm{J}$ Clin Periodontol. 1984;11:145-58.

6. Gittins DI, Bethell D, Schiffrin DJ, Nichols RJ. A nanometre-scale electronic switch consisting of a metal cluster and redox-addressable groups. Nature. 2000;408:67-69.

7. Wise $\mathrm{K}$ and Brasuel $\mathrm{M}$. The current state of engineered nanomaterials in consumer goods and waste streams: the need to develop nanoproperty-quantifiable sensors for monitoring engineered nanomaterials. NanotechnolSci Appl. 2011;4:73-86. 
8. Goldberg M, Langer R, Jia X. Nanostructured materials for applications in drug delivery and tissue engineering. $\mathrm{J}$ Biomater Sci Polym Ed. 2007;18:241-68.

9. Ramadan E, Borg Th, El Hawary Y.M and Saleh, N.M.: Formulation and evaluation of metronidazole bioadhesive matrices for treatment of periodontitis. Bull. Pharm. Sci., Assiut University 2010;33:79-94.

10. Martuscelli G1, Fiorellini JP, Crohin CC, Howell TH. The effect of interleukin-11 on the progression of ligature-induced periodontal disease in the beagle dog. J Periodontol. 2000;71:573-8.

11. Brunsvold MA and Mellonig JT. Bone grafts and periodontal regeneration. Periodontol 2000. 1993;1: 80-91.

12. Page RC and Schroeder HE. Periodontitis in man and other animal. A comparative review. Basel, Switzerland Karger 1982, 235-239.

13. Polson AM and Caton J. Factors influencing periodontal repair and regeneration. J Periodontol. 1982;53: 617-625.

14. Sigurdsson TJ, Lee MB, Kubota K, et al. Periodontal repair in dogs: recombinant human bone morphogenetic protein-2 significantly enhances periodontal regeneration. J Periodontol.1995;66: 131-8.

15. Kim CK, Cho KS, Choi SH, et al. Periodontal repair in dogs: effect of allogenic freeze-dried demineralized bone matrix implants on alveolar bone and cementum regeneration. J Periodontol. 1998;69 26-33.

16. Choi SH, Kim CK, Cho KS, et al. Effect of recombinant human bone morphogenetic protein-2/absorbable collagen sponge (rhBMP-2/ACS) on healing in 3-wall intrabony defects in dogs. J Periodontol. 73: 2002, 63-72.

17. Barrera DA, Zylstra E, Lansbury PT, Langer R. Synthesis and RGD peptide modification of a new biodegradable copolymer: poly (lactic acid-co-lysine). J Am Chem Soc 1993; 115: 11010-11011.

18. Esmaeili F, Hosseini-Nasr M, Rad-Malekshahi M, Samadi N, Atyabi F, Dinarvand R.: Preparation and antibacterial activity evaluation of rifampicin-loaded poly lactide-coglycolide nanoparticles. Nanomedicine 2007; 3: 161-167.

19. Hou Z, Zhou C, Luo Y, Zhan C, Wang Y, Xie L, et al. PLA nanoparticles loaded with an active lactone form of hydroxycamptothecin: development, optimization, and in vitro-in vivo evaluation in mice bearing H22 solid tumor. Drug Development Research 2011; 72:337-345.

20. Yadav KS, Chuttani K, Mishra AK, Sawant KK.: Long circulating nanoparticles of etoposide using PLGA-MPEG and PLGA-pluronic block copolymers: characterization, drug-release, blood-clearance, and biodistribution studies. Drug Development Research 2010;71: 228-239.

21. Pillai RR, Somayaji SN, Rabinovich M, Hudson MC, Gonsalves KE. Nafcillin-loaded PLGA nanoparticles for treatment of osteomyelitis. Biomed Mater. 2008;3:034114.

22. Dodwad V, Vaish S, Mahajan A, Chhokra M. Local Drug Delivery in Periodontics: A Strategic Intervention. International Journal of Pharmacy and Pharmaceutical Sciences 2012;4:30-34.

23. Walker $\mathrm{CB}$. The acquisition of antibiotic resistance in the periodontal microflora. Periodontol 2000 1996;10:79-88.

24. Poulet PP, Duffaut D, Lodter JP. Metronidazole susceptibility testing of anaerobic bacteria associated with periodontal disease. J Clin Periodontol 1999;26:261-3.

25. Bahar H, Torun MM, Demirci M. Antimicrobial resistance and $\beta$-lactamase production of clinical isolates of Prevotella and Porphyromonas species. Chemotherapy 2005;51;9-14.

26. Gelperina S1, Kisich K, Iseman MD, Heifets L. The potential advantages of nanoparticle drug delivery systems in chemotherapy of tuberculosis. Am J Respir Crit Care Med. 2005;172:1487-90.

27. De Jong WH1, Borm PJ. Drug delivery and nanoparticles: applications and hazards. Int J Nanomedicine. 2008;3:133-49.

28. Singh R1, Lillard JW Jr. Nanoparticle-based targeted drug delivery. Exp Mol Pathol. 2009;86:215-23. 